\title{
La competencia Aprender a Aprender en el marco de las competencias transversales clave de los Objetivos del Desarrollo Sostenible en las Universidades
}

\section{Victoria Vázquez Verdera ${ }^{a}$, Piedad Sahuquillo Mateo ${ }^{\mathrm{b},}$ Irene Verde Peleato ${ }^{\mathrm{c}}$, Verónica Riquelme Soto ${ }^{d}$}

${ }^{a}$ Victoria Vázquez Verdera (Universidad de Valencia, toya.vazquez@uv.es), ${ }^{\text {b }}$ Piedad Sahuquillo Mateo (Universidad de Valencia, piedad.sahuquillo@uv.es), ${ }^{\mathrm{C}}$ Irene Verde Peleato (Universidad de Valencia, irene.verde@uv.es) y ${ }^{\mathrm{d}}$ Verónica Riquelme Soto (Universidad de Valencia, veronica.riquelme@uv.es).

\begin{abstract}
The communication offers the possibilities of educational innovation that are raised from the research carried out to deepen the development of the competence learning to learn in university contexts. The results of the discussion groups are analyzed through the prism of the transversal key competences that are proposed to help achieve the Objectives of Sustainable Development through teaching. It ends with a few brief conclusions.
\end{abstract}

Keywords: competences, Objetives for Sustainable Development, Curricular Sustainability, learning to learn

\begin{abstract}
Resumen
La comunicación ofrece las posibilidades de innovación educativa que se plantean a partir de la investigación realizada para profundizar en el desarrollo de la competencia aprender a aprender en los contextos universitarios. Se analizan los resultados de los grupos de discusión a través del prisma de las competencias transversales clave que se proponen para ayudar a alcanzar los Objetivos de Desarrollo Sostenible a través de la docencia. Se finaliza con unas breves conclusiones.
\end{abstract}

Palabras clave: competencias, Objetivos de Desarrollo Sostenible, sostenibilización curricular, aprender a aprender. 
La competencia Aprender a Aprender en el marco de las competencias transversales clave de los Objetivos del Desarrollo Sostenible en las universidades

\section{Introducción}

En el documento Publicado en 2017 por la Organización de las Naciones Unidas para la Educación la Ciencia y la Cultura (UNESCO), "Educación para los Objetivos del Desarrollo Sostenible. Objetivos de aprendizaje" se proponen ocho competencias transversales clave para incorporar el espíritu de la Agenda 2030 en la docencia universitaria (UNESCO, 2017, p. 10):

- "Competencia de pensamiento sistémico: las habilidades para reconocer y comprender las relaciones; para analizar los sistemas complejos; para pensar cómo están integrados los sistemas dentro de los distintos dominios y escalas; y para lidiar con la incertidumbre

- Competencia de anticipación: las habilidades para comprender y evaluar múltiples escenarios futuros - el posible, el probable y el deseable; para crear visiones propias de futuro; para aplicar el principio de precaución; para evaluar las consecuencias de las acciones; y para lidiar con los riesgos y los cambios

- Competencia normativa: las habilidades para comprender y reflexionar sobre las normas y valores que subyacen en nuestras acciones; y para negociar los valores, principios, objetivos y metas de sostenibilidad en un contexto de conflictos de intereses y concesiones mutuas, conocimiento incierto y contradicciones

- Competencia estratégica: las habilidades para desarrollar e implementar de forma colectiva acciones innovadoras que fomenten la sostenibilidad a nivel local y más allá

- Competencia de colaboración: las habilidades para aprender de otros; para comprender y respetar las necesidades, perspectivas y acciones de otros (empatía); para comprender, identificarse y ser sensibles con otros (liderazgo empático); para abordar conflictos en grupo; y para facilitar la resolución de problemas colaborativa y participativa

- Competencia de pensamiento crítico: la habilidad para cuestionar normas, prácticas y opiniones; para reflexionar sobre los valores, percepciones y acciones propias; y para adoptar una postura en el discurso de la sostenibilidad

- Competencia de autoconciencia: la habilidad para reflexionar sobre el rol que cada uno tiene en la comunidad local y en la sociedad (mundial); de evaluar de forma constante e impulsar las acciones que uno mismo realiza; y de lidiar con los sentimientos y deseos personales. 
Victoria Vázquez Verdera, Piedad Sahuquillo Mateo, Irene Verde Peleato y Verónica Riquelme Soto

- Competencia integrada de resolución de problemas: la habilidad general para aplicar distintos marcos de resolución de problemas a problemas de sostenibilidad complejos e idear opciones de solución equitativa que fomenten el desarrollo sostenible, integrando las competencias antes mencionadas".

La competencia de Aprender a Aprender es clave para que la formación recibida en la universidad se alinee con las competencias transversales identificadas por la UNESCO (2015) para lograr transformar nuestro mundo: la Agenda 2030 para el Desarrollo sostenible. Las universidades tenemos la responsabilidad de comprometernos con el futuro de nuestro estudiantado, no podemos robarles su futuro. Para frenar la crisis planetaria las universidades necesitamos reinvertarnos a nosotras mismas porque ya no podemos hacer las cosas "como siempre". Es preciso aprender a aprender a manejarnos con la incertidumbre actual y la necesidad de tomar decisiones colaborativas a partir de la reflexión crítica y sistémica, de modo que seamos capaces de gestionar los propios aprendizajes y controlar los procesos en pro del bienestar personal y comunitario.

En relación a ello, Gargallo et al. (2015, p. 231) señalan la importancia del modelo centrado en el aprendizaje/centrado en el alumno para el desarrollo y consecución de la competencia de Aprender a Aprender. Del mismo modo, dichos autores y autoras plantean que el modelo centrado en el aprendizaje ha ido creciendo en importancia en la literatura y en la investigación sobre educación superior.

En suma, las competencias transversales que señala la UNESCO, la necesidad de promoción de la competencia de Aprender a Aprender en el alumnado, junto con el cambio de paradigma en la metodología de aprendizaje, pasando del modelo centrado en la enseñanza al modelo centrado en el aprendizaje, permiten los siguientes aspectos:

Promover el aprendizaje del alumno, su autonomía y sus habilidades metacognitivas de autorregulación. A su vez, se otorga relevancia a la incorporación de la enseñanza innovadora —con diferentes métodos: trabajo cooperativo, aprendizaje basado en problemas, desarrollo de proyectos, enseñanza de aprendizaje autorregulado, etccompatibles con la metodología expositiva de calidad. El uso de una evaluación significativa, utilizando diferentes fuentes de recogida de información, que devuelve feedback a los alumnos y les da la oportunidad de participar en el proceso, al igual que un currículum flexible, dándole posibilidades de elegir rutas alternativas de aprendizaje. El papel del profesor es el de mediador y articulador de buenos entornos y experiencias de aprendizaje. (Gargallo et al., 2017, p. 164) 
La competencia Aprender a Aprender en el marco de las competencias transversales clave de los Objetivos del Desarrollo Sostenible en las universidades

\section{Objetivos}

Proponer experiencias docentes para la mejora de la calidad de los procesos de enseñanza-aprendizaje desarrollados en el ámbito de la educación a consecuencia de los resultados de la investigación que estamos desarrollando (Diseño operativo de la competencia 'Aprender a Aprender' para los grados universitarios. Instrumentos de evaluación y propuestas para la enseñanza. EDU2017-83284-R).

Tras el proceso de recogida de datos sobre la percepción de quienes están estudiando, de quienes están ejerciendo las profesiones y de quienes les están empleando con respecto a la enseñanza de la competencia Aprender a Aprender, proponer una serie de mejoras en relación a las necesidades de aprendizaje que existen en relación a la sostenibilidad humana y ambiental.

\section{Método}

Se llevó a cabo seis grupos de discusión moderados por el grupo investigador, y en el que participaron los tres colectivos: profesorado, alumnado, empleadores y profesionales y las tres áreas de conocimiento: ciencias de la salud, ingenierías y ciencias de la educación La cuestión planteada fue la siguiente:

Desde tu punto de vista, y en relación con el "aprender a aprender", con la necesidad de seguir aprendiendo, ¿qué cualidades, habilidades, etc. debe adquirir o perfeccionar una persona durante sus estudios de grado en la universidad para convertirse en un profesional competente y responsable?

\section{Desarrollo de la innovación}

Diseñar los procesos de enseñanza-aprendizaje por competencias exige ir más allá de los formatos disciplinares convencionales para ofrecer experiencias de aprendizaje que se concretan en resultados de aprendizaje en el o la estudiante.

En coherencia con los referentes axiológicos de los Objetivos para el Desarrollo Sostenible, se plantea la necesidad de proponer innovaciones didácticas para que los resultados de aprendizaje consigan que los futuros y las futuras profesionales sean agentes de transformación, piensen de forma sistémica y crítica, se responsabilicen con el bienestar del planeta y las personas que lo habitan y logren la transformación de forma colaborativa. Las innovaciones concretas a desarrollar se ajustan a las demandas resultantes delproceso investigador. 


\section{Resultados}

De los grupos de discusión recogimos la necesidad de implementar innovaciones docentes para dar respuesta a las necesidades que planteaban:

Empleadora, educación:

"Entonces necesitamos personas arriesgadas, personas flexibles, personas creativas sobre todo y que trabajen en equipo... Vamos, ¡eso es fundamental! Pero yo creo que la flexibilidad es una de las cosas más importantes porque somos, pretendemos, que nuestros alumnos sean muy creativos, que nos den lluvia de ideas ...que sean...y luego nosotros somos como muy recalcitrantes en este tema, ¿no? es decir, nos da mucho miedo adaptarnos...entonces, si no te lanzas en educación, nunca puede haber un cambio y somos muy reacios al cambio. La gente que no tema al cambio. Es que el cambio es necesario, el cambio es la vida, todos los días hay un cambio, entonces te tienes que adaptar, y, además, eso es una señal de inteligencia, o sea las personas que más rápidamente se adaptan a un cambio, más inteligentes emocionalmente son...”

\section{Estudiante. Salud:}

"De hecho, en las simulaciones que hacemos aquí en la Facultad es muy importante tener esa habilidad de saber... de prepararte a lo que pueda venir; y eso es una cosa que no la sabemos dominar, por lo menos, a día de hoy como nos gustaría o como pretenden que la tengamos dominada; y es que eso es, básicamente, no un problema de la Facultad, ni de los profesores, ni de los estudiantes; también es un problema de los profesores, por ejemplo de los PACs, que no nos dejan “.

\section{Profesora. Salud:}

“que desarrollaran esa capacidad crítica: una opinión, un análisis, observación... que sean capaces de discernir entre qué es positivo y qué es negativo para él. Para eso yo creo que un alumno tiene que dejar de... ser un elemento pasivo para convertirse en un elemento activo y ser constructor de su propio aprendizaje. Para que uno construya aprendizaje, eh... al final tiene que... Para dar esa vuelta tenemos que acercar lo que está viendo en... o el contenido teórico que está viendo a la realidad. Resolviendo problemas reales".

Profesor. Ingenierias:

"Experiencia que más me enriquece a mi y a ellos también es el grupo de 4 alumnos que hacen un proyecto, auditoria ambiental, mi laboratorio es el territorio, o me los llevo al campo o tengo que hacer fotografías o forn imaginación..., así que yo todos los años 
La competencia Aprender a Aprender en el marco de las competencias transversales clave de los

Objetivos del Desarrollo Sostenible en las universidades

concierto con un ayuntamiento una actividad. Me los llevo al campo, les enseño a tomar datos, los chavales no saben qué han de tomar, no saben en que fijarse, conversamos sobre lo que vemos, interpretar, etc. El resultado lo exponen ante el/la alcalde, concejales, colectivo de 20 personas se lo explico el primer día y alumnos se desmatriculan, otros vienen, ... les impone la actividad, pero les viene muy bien porque ellos saben hacer cálculos, pero no saben relacionar. Y les enseñamos el razonamiento crítico, a que los valores que obtienen siempre significan algo y es lo que intentamos trabajar con ellos. En grupos pequeños por supuesto".

Estudiante. Ingenierías:

"Hay que saber diferenciar porque funciona las cosas y donde enfocarlas, que cambiar, porque ok, voy a tomar un modelo, pero no lo tomen a puño y letra, tómalo léelo, analízalo y de dónde salen las cosas, es igual en el trabajo, te presentan mucha información, sales al campo y dices hoy voy aprender, tienes que salir con una actitud de esponja, absorber todos los conocimientos y tratar al final de cómo te las arreglas y como a mí siempre me dijeron cuando empecé sin nada de experiencia, ante la duda, claro no es que me preguntes cada cinco minutos, pero ante la duda siempre apóyate con una persona, no es que en ese momento claro tú no sabes pero ya en ese momento que preguntes ya sabes todo.

No preguntar las cosas una por una pero, acumulas todo lo que vas a preguntar y con las dudas y todo con una buena capacidad de comunicación, claro no es presentar darle la duda, es presentarle que tú tienes algo que no entiendes pero no se lo estás diciendo en modo de duda, sino más en modo de conversación, saber desarrollar esa conversación, saber desarrollar ese hilo saber que solución podría darle esa persona y que salida podrías darle tu que no fuera la misma que él le diera. Porque hoy en día los empleadores no buscan a personas a estudiantes o a personas recién graduadas porque estudiaron lo mismo que tú".

\section{Conclusiones}

Concluimos con la necesidad de suscribir el compromiso de la Comisión Sectorial CRUE Sostenibilidad, con más de diez años de trayectoria y resultados probados tanto en el terreno de la ambientalización de la gestión institucional como en la sostenibilización currícular:

La educación debe, por tanto: Tener un enfoque integrado sobre los conocimientos, las actitudes, las habilidades y los valores en la enseñanza; Promover el trabajo en equipos multidisciplinares; Estimular la creatividad y el pensamiento crítico; Fomentar la reflexión y el autoaprendizaje; Reforzar el pensamiento sistémico y un enfoque holístico; Formar personas participativas y pro-activas que sean capaces de 
tomar decisiones responsables; Adquirir conciencia de los desafíos que plantea la globalización; Promover el respeto a la diversidad y la cultura de la paz (CRUE, 2012, p. 3).

Cabe esperar que en los próximos años, con motivo de las modificaciones de planes de estudio exigidas por los procesos de acreditación de las titulaciones universitarias, podamos asistir a la inclusión generalizada de la sostenibilidad como criterio de calidad de los centros universitarios (Murga, 2017). Para transitar hacia la sostenibilidad es necesario descentrarse de los procesos que actualmente dirigen nuestras pautas de consumo y producción científica y cultural, y que tienen un impacto directo en nuestros planteamientos docentes.

En los grupos de discusión se identifica que las universidades deben preparar profesionales que sean capaces de utilizar sus conocimientos, no sólo en un contexto científico, sino también para responder a las necesidades sociales y ambientales. Las innovaciones docentes resultantes del proceso investigador se ajustan a las demandas de los tres colectivos estudiados. Se reconoce la importancia de establecer mecanismos para mejorar la formación que recibirán los y las futuras profesionales, de manera que sean capaces de afrontar los retos de aprendizaje que plantea la situación global actual en cuanto al impacto de la desigualdad de género, la pobreza, la dependencia enérgetica y su impacto en los derechos humanos, el cambio climático, los residuos, entre otras.

Necesitamos cambios profundos en el modelo dominante para revertir la insostenibilidad del sistema actual y una formación universitaria que permita a las nuevas generaciones ejercer su compromiso profesional en pro de una cultura económica, política y técnica que ponga en el centro la vida de las personas y el planeta que habitamos.

\section{Referencias}

CRUE. (2012). Directrices para la introducción de la Sostenibilidad en el Curriculum. Disponible en: https://bit.ly/2EL3GRz

Gargallo, B., Garfella, P. R., Sahuquillo, P. y Verde, I. (2015). Métodos centrados en el aprendizaje, estrategias y enfoques de aprendizaje en estudiantes universitarios. Revista de Educación, (370), 229-241. Disponible en: https://bit.ly/2W0fPYB

Gargallo, B., Pérez, C., Jiménez, M. A., Martínez, N. y Giménez, J. A. (2017). Métodos centrados en el aprendizaje, implicación del alumno y percepción del contexto de 
La competencia Aprender a Aprender en el marco de las competencias transversales clave de los Objetivos del Desarrollo Sostenible en las universidades aprendizaje en estudiantes universitarios. Educación XXI, Vol. 22 (2), 161- 187. Disponible en: https://bit.ly/2YWFwuT

Murga, M.A. (2017). Universidades en transición. Hacia una transformación institucional orientada al logro de la sostenibilidad. Revista Iberoamericana de Educación, Vol. 73 (1), pp. 61-84. Disponible en: https://bit.ly/2WxtT06

UNESCO. (2018). Educación para los Objetivos del Desarrollo Sostenible. Objetivos de aprendizaje. París: UNESCO. Disponible en: https://bit.ly/2IHAwHs

ONU. (2016). Transformar nuestro mundo: Agenda 2030 para el Desarrollo Sostenible. Disponible en: https://bit.ly/2APa8W3 